(49\%; $P=0.002)$. Logistic regression analysis indicated that siblings of an individual with LMD had a 3.6 (95\% Cl 1.7-7.1) recurrent risk ratio of presenting with $L M D$.

In a further prospective, nested case-control study, initially healthy siblings of patients with CAD were studied; 79 siblings had a coronary event during follow-up, while 235 remained event-free (controls). A positive sibling history of LMD was present for $13.9 \%$ of patients with an incident event, versus $6.4 \%$ of controls $(P=0.036)$. The risk of experiencing a future coronary event was 2.5 to 3 -fold higher in siblings of patients with LMD than in siblings of patients with other manifestations of CAD. These findings indicate the potential for intensified primary prevention of coronary events in selected families.

Original article Fischer M et al. (2007) Familial aggregation of left main coronary artery disease and future risk of coronary events in asymptomatic siblings of affected patients. Eur Heart J 28: 2432-2437

\section{Measuring proBNP identifies dyspnea caused by cardiac disease}

Patients commonly present with dyspnea, but determining whether this symptom has a cardiac or a pulmonary cause is difficult. Emergency measurement of natriuretic peptides can show whether acute dyspnea is associated with an acute cardiac condition, but it is not known whether this test is relevant for use in members of the general population who have chronic dyspnea.

Mogelvang et al. carried out a large populationbased study to evaluate pro-brain naturiuretic peptide (proBNP) as a possible biomarker for cardiac disorders in people with chronic dyspnea. The authors measured plasma proBNP levels and investigated the cause of dyspnea, as determined by spirometry, oxygen saturation and echocardiography.

Of the 2,929 study participants, 959 reported dyspnea. As expected, plasma proBNP concentrations were higher in patients with dyspnea than in those without. Patients with left ventricular hypertrophy, systolic dysfunction or both had a mean 2.6-fold elevation in proBNP levels when compared with patients with pulmonary dysfunction $(P<0.001)$.
After employing a multivariate regression analysis, the authors concluded that an actual plasma proBNP concentration below half of the expected value for the age and sex of a patient with dyspnea rules out left ventricular systolic and diastolic dysfunction. They suggest that plasma proBNP levels could form the basis of a highly sensitive and specific test to identify the underlying cause of chronic dyspnea.

Original article Mogelvang R et al. (2007) Discriminating between cardiac and pulmonary dysfunction in the general population with dyspnea by plasma pro-B-type natriuretic peptide. J Am Coll Cardiol 50: 1694-1701

\section{Cardiac troponin levels predict death in patients stabilized after acute coronary syndromes}

Elevated levels of cardiac troponin are associated with an increased risk of adverse cardiovascular events in patients with non-ST-segment elevation acute coronary syndromes (ACS), but might not be detectable in patients with stable coronary artery disease. Eggers et al. studied 1,092 patients who had been stabilized after an ACS episode, to investigate whether slightly raised troponin levels could augment risk assessment for cardiac-related mortality.

During a 5-year follow-up as part of the FRagmin and Fast Revascularization during InStability in Coronary artery disease (FRISC-II) trial, cardiac troponin I levels were measured at 6 weeks, 3 months and 6 months after enrollment, using the sensitive Access AccuTnl assay.

Almost half of the patients (48\%) had elevated troponin levels at the 6 -week follow-up visit: the proportion fell to $36 \%$ at 6 months. Troponin levels were elevated at all three time points in $26 \%$ of patients: these patients were generally older and had a variety of other cardiovascular disease risk factors. At 5 years, $106(10 \%)$ patients had died. The lowest cutoff point for troponin elevation $(0.01 \mathrm{ng} / \mathrm{l})$ was found to be an independent predictor of mortality, even after adjustment for other cardiovascular risk factors. This finding was observed whether patients received invasive or noninvasive treatment.

The authors recommend careful monitoring of cardiac troponin levels in patients with ACS 